\title{
Bases conceituais para a aplicação de biomonitoramento em programas de avaliação da qualidade da água de rios
}

\author{
Conceptual basis for the application \\ of biomonitoring on stream water \\ quality programs
}

Daniel Forsin Buss 1

Darcílio Fernandes Baptista 1

Jorge Luiz Nessimian 2

\footnotetext{
1 Laboratório de Avaliação e Promoção da Saúde Ambiental, Departamento de Biologia, Instituto Oswaldo Cruz, Fundação Oswaldo Cruz. Av. Brasil 4365, Rio de Janeiro, RJ 21045-900, Brasil. buss@centroin.com.br 2 Laboratório de Entomologia, Departamento de Zoologia, Centro de Ciências da Saúde, Universidade Federal do Rio de Janeiro. C. P. 68044, Rio de Janeiro, $R J$ 21944-970, Brasil.
}

\begin{abstract}
Biomonitoring is defined as the systematic use of biological responses to assess environmental changes, usually anthropogenic impacts. In this article we present the conceptual basis and a brief history of biomonitoring as an assessment tool for environmental health. Considering the drawbacks of physical and chemical parameters to assess environmental quality, we pinpoint the need to integrate these analyses with information provided by biological monitoring. The application of biomonitoring in Brazil would help watershed managers and policymakers to reduce costs, increase the efficiency of analyses, and simplify the results, allowing community participation through volunteer monitoring programs.
\end{abstract}

Key words Environmental Monitoring; Water Monitoring; Water Quality; Rivers; Natural Resources Management

Resumo Biomonitoramento pode ser definido como o uso sistemático das respostas de organismos vivos para avaliar as mudanças ocorridas no ambiente, geralmente causadas por ações antropogênicas. Neste artigo são apresentadas as bases conceituais e um breve histórico da utilização do biomonitoramento como ferramenta de avaliação da saúde dos ecossistemas de rios. Busca ainda fornecer subsídios para uma análise integrada da qualidade da água, pois as metodologias tradicionais de avaliação, baseadas em características físicas, químicas e bacteriológicas, não são suficientes para atender aos usos múltiplos da água, sendo particularmente deficientes na avaliação da qualidade estética, de recreação e ecológica do ambiente. Visando colaborar com os gestores de bacias hidrográficas, a aplicação do biomonitoramento no Brasil contribui para a redução de custos, o aumento da eficiência de análise e a simplificação dos resultados, permitindo a participação comunitária por intermédio de grupos de voluntários.

Palavras-chave Monitoramento Ambiental; Monitoramento da Água; Qualidade da Água; Rios; Gestão de Recursos Naturais 


\section{Introdução}

O primeiro passo para a resolução dos problemas sócio-ambientais gerados pela má gestão dos recursos hídricos é o desenvolvimento de metodologias de diagnóstico eficientes. Segundo documento da Organização das Nações Unidas (ONU), Agenda 21 (CNUMAD, 1992:333), “ $a$ utilização da água deve ter como prioridades a satisfação das necessidades básicas e a preservação dos ecossistemas." No capítulo 18 desse documento é sugerido que a proteção da qualidade e do abastecimento dos recursos hídricos seja feita a partir da aplicação de critérios integrados para o desenvolvimento, o manejo e o uso dos recursos hídricos.

A discussão sobre a importância da utilização de critérios integrados não é recente. Desde a década de 1970, pesquisadores e gestores de recursos hídricos da Europa Ocidental e da América do Norte (Armitage, 1995; Cairns Jr. \& Pratt, 1993; Pratt \& Coler, 1976) argumentam que as metodologias tradicionais de classificação de águas, baseadas em características físicas, químicas e bacteriológicas, não são suficientes para atender aos usos múltiplos da água, sendo particularmente deficientes na avaliação da qualidade estética, de recreação e ecológica do ambiente. Isso pode ser atingido com uma análise integrada da qualidade da água, ou seja, considerando não apenas as metodologias tradicionais de avaliação, mas os aspectos biológicos do sistema (Barbosa, 1994; Metcalfe, 1989; Rosenberg \& Resh, 1993).

Para analisar os aspectos biológicos dos ecossistemas, duas metodologias vêm sendo utilizadas. Os métodos “bottom-up" utilizam fundamentalmente dados de laboratório por meio de experimentação em sistemas simples com subseqüente extrapolação para sistemas mais complexos. A metodologia "top-down" avalia, em nível macro, os impactos ambientais por meio da medição da alteração da organização estrutural e funcional das comunidades biológicas ou dos ecossistemas.

Os testes da metodologia bottom-up são realizados, em geral, com base nas respostas de organismos aquáticos a estressores específicos. Nesses casos, são usados como indicadores alterações bioquímicas (enzimáticas ou genéticas, por exemplo), fisiológicas (regulação iônica), comportamentais, metabólicas e do ciclo de vida (Boudou \& Ribeyre, 1997; Buikema \& Voshell, 1993; Calow, 1993; Pivetta et al., 2001; Rosenberg \& Resh, 1993). O uso das respostas fisiológicas em avaliações de laboratório é genericamente conhecido como testes toxicológicos. Dentre eles destacam-se os bioensaios de reação aguda, especializados na determinação de poluição letal e subletal de substâncias sintéticas ou naturais de origem mineral, animal ou vegetal (Ewell et al., 1986). A avaliação em laboratório envolve ainda a análise da exposição crônica, conferindo os efeitos deletérios quanto a genotoxicidade, carcinogenicidade e mutagenicidade (Reynoldson \& MetcalfeSmith, 1992).

Por sua vez, as metodologias top-down se provaram viáveis em muitos casos e representam o caminho mais rápido e prático para o manejo dos ecossistemas. Com esses métodos ganha-se controle e velocidade na reação em testes de toxicidade, mas perde-se contato com a realidade e aplicabilidade ao ambiente (Moulton, 1998). Assim, para a análise da qualidade de águas é vantajoso o uso de ferramentas topdown, por avaliarem eficientemente:

- a perda real da diversidade de espécies, em vez de avaliarem os efeitos indiretos dos agentes estressores;

- o efeito sinergético das alterações antropogênicas ocorridas na bacia hidrográfica (por exemplo, a soma dos efeitos do desmatamento, da entrada de pesticidas e de efluentes domésticos);

- a qualidade da água por métodos relativamente simples e de baixo custo;

- o impacto de espécies exóticas sobre a fauna e flora locais; e

- a integridade ecológica dos ecossistemas aquáticos.

O objetivo deste artigo é apresentar as bases conceituais da utilização do biomonitoramento como ferramenta de avaliação da saúde dos ecossistemas de rios, fornecendo subsídios para uma análise integrada da qualidade da água e apresentando perspectivas para sua aplicação no Brasil.

\section{Bases conceituais do biomonitoramento}

O uso de parâmetros biológicos para medir a qualidade da água se baseia nas respostas dos organismos em relação ao meio onde vivem. Como os rios estão sujeitos a inúmeras perturbações, a biota aquática reage a esses estímulos, sejam eles naturais ou antropogênicos. A habilidade de proteger os ecossistemas depende da capacidade de distinguir os efeitos das ações humanas das variações naturais, buscando categorizar a influência das ações humanas sobre os sistemas biológicos (Cairns Jr. et al., 1993). Nesse contexto, a definição de biomonitoramento mais aceita é o uso sistemático das respostas de organismos vivos para 
avaliar as mudanças ocorridas no ambiente, geralmente causadas por ações antropogênicas (Matthews et al., 1982).

A idéia de que espécies podem ser usadas para indicar certas condições ambientais tem sido verificada com bastante freqüência ao longo da história. Um exemplo ocorreu durante a Revolução Industrial (Século XIX), quando canários eram colocados dentro de minas de carvão para monitorar a qualidade do ar. Caso o canário sofresse alguma alteração desfavorável, causada por altas concentrações de monóxido de carbono, as pessoas eram imediatamente retiradas do local, evitando possíveis danos à saúde (Cairns Jr. \& Pratt, 1993).

O uso das respostas dos organismos é a base dos índices biológicos. Bioindicadores são espécies escolhidas por sua sensibilidade ou tolerância a vários parâmetros, como poluição orgânica ou outros tipos de poluentes (Washington, 1984). O termo "resposta biológica" se refere ao conjunto de reações de um indivíduo ou uma comunidade em relação a um estímulo ou a um conjunto de estímulos (Armitage, 1995). Por estímulos entendemos algo que induza uma reação do indivíduo que possa ser percebida e medida na população ou na comunidade.

Segundo Metcalfe (1989), o uso das respostas biológicas como indicadores de degradação ambiental é vantajoso em relação às medidas físicas e químicas da água, pois estas registram apenas o momento em que foram coletadas, como uma fotografia do rio, necessitando assim de um grande número de análises para a realização de um monitoramento temporal eficiente. Outra desvantagem é que, se forem feitas longe da fonte poluente, as medições químicas não serão capazes de detectar perturbações sutis sobre o ecossistema (Pratt \& Coler, 1976).

Por sua vez, os organismos integram as condições ambientais durante toda a sua vida, permitindo que a avaliação biológica seja utilizada com bastante eficiência na detecção tanto de ondas tóxicas intermitentes agudas quanto de lançamentos crônicos contínuos (De Pauw \& Vanhooren, 1983). Além disso, as metodologias biológicas são bastante eficazes na avaliação de poluição não pontual (difusa), tendo, portanto, grande valor para avaliações em escala regional (Pratt \& Coler, 1976).

Mesmo em casos de lançamentos contínuos dentro das normas estabelecidas por lei, o uso da biota aquática é uma importante ferramenta na avaliação da qualidade da água. Isso se deve a um processo natural denominado biomagnificação, que é a transmissão de compostos que não são metabolizados ou excreta- dos pelos organismos para o nível superior da cadeia trófica. Em alguns casos esses compostos podem ser tóxicos se acumulados, como no caso de metais pesados e de pesticidas organoclorados. Portanto, mesmo estando dentro das normas legais de lançamento, esses efluentes podem estar degradando as inter-relações biológicas, extinguindo espécies e gerando problemas de qualidade de vida para as populações que utilizam aquele recurso.

Os indicadores biológicos são muito úteis por sua especificidade em relação a certos tipos de impacto, já que inúmeras espécies são comprovadamente sensíveis a um tipo de poluente, mas tolerantes a outros (Washington, 1984). Assim, índices podem ser criados especificamente para detectar derramamento de óleo, poluição orgânica, alteração de $\mathrm{pH}$ da água, lançamento de pesticidas, entre outros.

\section{Definição de indicadores biológicos}

Segundo Johnson et al. (1993), um indicador biológico "ideal" deve possuir as seguintes características:

- ser taxonomicamente bem definido e facilmente reconhecível por não-especialistas;

- apresentar distribuição geográfica ampla;

- ser abundante ou de fácil coleta;

- ter baixa variabilidade genética e ecológica;

- preferencialmente possuir tamanho grande;

- apresentar baixa mobilidade e longo ciclo

de vida;

- dispor de características ecológicas bem conhecidas; e

- ter possibilidade de uso em estudos em laboratório.

A primeira abordagem visando à determinação de indicadores biológicos da qualidade das águas, com bases científicas, foi feita com bactérias, fungos e protozoários na Alemanha por Kolkwitz \& Marsson (1909). Como praticamente qualquer grupo pode ser utilizado em programas de monitoramento, foram desenvolvidas metodologias de avaliação para macrófitas aquáticas (Best, 1990; Haslam, 1982), peixes e macroinvertebrados. A utilização da comunidade de peixes com essa finalidade tem sido extensamente implantada, principalmente nos Estados Unidos (Cairns Jr. \& van der Schalie, 1980; Fausch et al., 1990; Karr, 1981; Karr et al., 1986), inclusive com proposta de uso em programas em todo o país (Fausch et al., 1984; Plafkin et al., 1989).

Apesar do desenvolvimento de metodologias de avaliação com diversos organismos, vários autores afirmam que o grupo de macroin- 
vertebrados bentônicos é o mais testado e utilizado (Barbour et al., 1999; Kerans \& Karr, 1994; Rosenberg \& Resh, 1993). Segundo Plafkin et al. (1989), essas comunidades têm sido amplamente utilizadas por uma série de razões:

- são ubíquos, podendo responder a perturbações em todos os ambientes aquáticos e em todos os períodos;

- o grande número de espécies oferece um amplo espectro de respostas;

- mesmo em rios de pequenas dimensões, a fauna pode ser extremamente rica;

- a natureza relativamente sedentária de várias espécies permite uma análise espacial eficiente dos efeitos das perturbações;

- apresenta metodologias de coleta simples e de baixo custo, que não afetam adversamente o ambiente; $\mathrm{e}$

- são relativamente fáceis de identificar segundo as metodologias existentes.

\section{Evolução do biomonitoramento em ecossistemas aquáticos}

O sistema sapróbico de Kolkwitz \& Marsson (1909) enfatizava que a abundância de organismos nas áreas poluídas ocorria por características fisiológicas e comportamentais que lhes permitia tolerar tais condições. Assim, localidades onde eram encontrados números elevados desses organismos eram classificadas como poluídas. No entanto, como organismos tolerantes à poluição são freqüentemente encontrados também em áreas íntegras, o desaparecimento ou uma redução considerável na abundância relativa dos chamados grupos sensíveis passou a ser considerado um fator importante em estudos posteriores, embora haja críticas sobre o real valor da "ausência" de uma espécie (Cairns Jr. \& Pratt, 1993).

A partir dessa primeira tentativa de classificação de ambientes com base na fauna local surgiram diversas metodologias, que podem ser divididas em três grandes grupos: os índices bióticos, os modelos de predição de impacto e os protocolos de avaliação rápida.

\section{Índices bióticos}

No final da década de 1960, iniciaram-se esforços conjuntos na Europa para testar a aplicabilidade do sistema sapróbico. A maioria dos países, à exceção da Alemanha e da Holanda, decidiu rejeitar esses métodos e passou a utilizar metodologias de avaliação representadas por índices bióticos de macroinvertebrados bentônicos, que consistiam em atribuir um "valor" (score) para cada espécie com base em sua tolerância ao impacto (Metcalfe, 1989). Diversos índices bióticos surgiram e foram testados desde então (ver revisões em Cairns Jr. \& Pratt, 1993; Metcalfe, 1989), mas um índice em especial ganhou destaque, o BMWP.

Em 1976, foi criado na Grã-Bretanha um grupo de trabalho para discutir e sintetizar o conhecimento sobre os índices, originando o sistema conhecido por Biological Monitoring Working Party score system (BMWP). Nos anos subseqüentes, o índice BMWP foi testado e revisto (ver histórico em Hawkes, 1997), e atualmente considera macroinvertebrados identificados ao nível taxonômico de família, com valores entre 1 e 10 atribuídos com base em sua sensibilidade a poluentes orgânicos. Famílias sensíveis a altos níveis de poluentes recebem valores mais altos, enquanto famílias tolerantes recebem valores mais baixos. Após o registro de ocorrência dos táxons em uma localidade, somam-se os valores referentes a cada família, obtendo-se um valor final para a localidade. Quanto maior esse valor, mais íntegra a localidade. A aplicação do índice ASPT (Average Score Per Taxon), que é a média dos valores de cada família encontrada (Balloch et al., 1976), tornou esse índice ainda mais eficiente (Armitage et al., 1983; Walley \& Fontama, 1998; Walley \& Hawkes, 1997).

Recentemente o índice tem ganho destaque ainda maior, pois vem sendo aplicado em programas nacionais de predição de impacto (Walley \& Hawkes, 1996; Wright, 1995).

\section{Modelos de predição de impacto}

No fim da década de 1980, Inglaterra e Austrália investiram na construção de modelos preditivos, RIVPACS (River Invertebrate Prediction And Classification System) e AusRivAS (Australian Rivers Assessment System), baseados inicialmente nos valores do BMWP.

A primeira etapa para a construção desses modelos foi o agrupamento, em 16 comunidades, de macroinvertebrados baseados em 268 trechos de rio "referência" (não poluídos), mediante análises multivariadas (Armitage et al., 1983). Por meio de análise discriminante múltipla, foram correlacionadas 28 variáveis ambientais a esses grupos de espécies (Wright et al., 1984). Assim, tem-se "comunidades esperadas" associadas a essas variáveis ambientais.

Para avaliar o grau de impacto de uma localidade "teste", analisam-se os parâmetros ambientais e a fauna de macroinvertebrados (comunidade observada). As variáveis ambientais determinam de qual grupamento de localida- 
des o local teste faz parte. Comparando as comunidades esperadas e observadas pode-se medir o grau de impacto da localidade (Moss et al., 1987).

Atualmente o RIVPACS III é o mais moderno e o principal instrumento de bioavaliação utilizado pelas autoridades do Reino Unido e da Austrália em seus programas nacionais de avaliação da qualidade da água em mais de 8 mil trechos de rios (Walley \& Hawkes, 1996; Wright et al., 1993).

\section{Protocolos de avaliação rápida \\ da qualidade da água (PAR)}

Enquanto nos países europeus predominaram as abordagens surgidas com base no índice de saprobidade, na América do Norte houve uma preferência por métodos de similaridade entre comunidades e de estatística multivariada, baseados primeiramente em uma classificação ambiental $a$ priori a partir de parâmetros físicos e químicos (Barbour et al., 1999).

O uso de macroinvertebrados nos programas de monitoramento da qualidade das águas nos Estados Unidos passou por duas importantes transições. Por volta de 1960, o uso preponderante era de abordagens qualitativas, baseadas no sistema de saprobidade. A primeira transição ocorreu na década de 1970, quando passou a ser enfatizada uma abordagem quantitativa para o cálculo de índices de diversidade. A segunda transição foi uma volta às abordagens qualitativas na implantação dos rapid assessment approaches ou protocolos de avaliação rápida (PAR) da qualidade da água (Resh \& Jackson, 1993).

Nos PAR, uma ou mais medidas bioindicadoras podem ser utilizadas. Essas medidas podem estar associadas a diferentes níveis hierárquicos de organização (espécie, populações ou comunidades) e podem ser divididas em cinco categorias: número de espécies (riqueza), enumerações (abundância dos grupos taxonômicos), diversidade e similaridade entre comunidades, medidas tróficas e índices bióticos.

Esses protocolos se baseiam em comparações entre locais "referência" (considerados controle por apresentarem excelentes condições de integridade ambiental) e as áreas a serem analisadas. Os países que utilizam essa ferramenta passaram a avaliar o conceito de ecorregião em seus programas de biomonitoramento. As ecorregiões são definidas por características fisiográficas como geologia, tipo de solo, vegetação natural potencial e uso da terra, partindo do princípio de que comunidades biológicas dentro de uma região homogênea são similares (Omernik, 1987; Whittier et al., 1988). A estrutura e a variabilidade natural das comunidades encontradas nas bacias hidrográficas de uma ecorregião podem ser calculadas, e as comunidades das áreas de referência podem servir como padrão para serem comparadas com outras localidades daquela ecorregião.

Segundo Resh \& Jackson (1993), esses protocolos são análogos ao uso de termômetros na avaliação da saúde humana, onde valores facilmente obtidos são comparados com o que se considera "normal" (por exemplo, a temperatura corporal humana de $37^{\circ} \mathrm{C}$ ). Esses autores apontam algumas questões relativas ao uso de métodos de avaliação: que medidas da população e/ou comunidade são biologicamente relevantes (os termômetros)? Quais são os limites normais com os quais estão sendo comparados (a temperatura normal do corpo)? Que limite do desvio da normalidade é um sinal de "doença"?

Em geral, pode-se dizer que não existe consenso entre os especialistas sobre o melhor modelo a ser aplicado. Cada método apresenta vantagens intrínsecas. Os índices bióticos podem ser aplicados por especialistas ou por pessoas não técnicas com treinamento em identificação taxonômica, são relativamente simples de serem analisados e sua implementação é a menos onerosa; sua aplicação, porém, depende de conhecimento prévio sobre o grau de sensibilidade dos organismos na região. Os defensores dos PAR argumentam que as respostas multimétricas são mais apropriadas do que as baseadas em uma métrica apenas (índices bióticos, por exemplo) e, portanto, mais robustas (Barbour et al., 1996). No entanto, para a aplicação dessa abordagem é necessário o conhecimento sobre estrutura e funcionamento das comunidades aquáticas, sendo fundamental o desenvolvimento de mais estudos descritivos para sua aplicação no Brasil. O RIVPACS tem se mostrado um modelo promissor por ser o único a apresentar característica preditiva de impacto. Entretanto, sua aplicação depende de um enorme esforço de coleta e análise na fase inicial, o que o tornaria inviável para uso em larga escala no Brasil neste momento.

A escolha da metodologia a ser utilizada em programas de biomonitoramento no Brasil depende, portanto, do atual estágio do conhecimento, que é bastante distinto regionalmente. 


\section{Desenvolvimento do monitoramento das águas no Brasil}

Embora o Brasil tenha sido representado na primeira reunião da Sociedade Internacional de Limnologia em 1922 (Esteves, 1988), o país não seguiu as tendências mundiais de avaliação e conservação desses sistemas. A esse fato procura-se atribuir algumas explicações, nenhuma delas convincente, como a abundância do recurso - o país possui a maior disponibilidade hídrica do planeta, com 13,8\% do deflúvio médio mundial (WRI, 1998) -, e o não-cumprimento da legislação, reconhecidamente uma das mais rígidas do mundo.

Do primeiro dispositivo legal voltado exclusivamente para os recursos hídricos, o Código das Águas (Decreto no 24.643 de 10 de julho de 1934 - Brasil, 1934), passando pela Resolução do Conselho Nacional do Meio Ambiente (CONAMA) no 20 de 1986 (Brasil, 1986), até a Lei das Águas (Lei 9.433/97 - Brasil, 1997), não se faz qualquer menção ao uso de biomonitoramento para avaliar a qualidade das águas.

O descaso com essa metodologia de avaliação ambiental fica evidente quando se analisa o documento Programa Monitore do Ministério do Meio Ambiente (MMA, 1998), que apresenta 65 projetos de "monitoramento da qualidade das águas” no Brasil. Desses, 59 são de águas doces, dos quais 42 em rios. Desse montante, 26 (61,9\%) compreendem apenas análises físicas, químicas e/ou bacteriológicas da água. Dos 16 programas de monitoramento restantes, que apresentam análises químicas e algum componente biológico, apenas quatro ainda estavam em funcionamento naquela data. Isso representa menos de $10 \%$ dos projetos de qualidade da água de rios, dos quais dois apresentam a vaga descrição "monitoramento de corpos d'água sem data prevista para finalização”.

Mesmo sem o devido apoio, alguns trabalhos têm abordado o tema, principalmente nas regiões sudeste e sul do País. Alguns exemplos são os de Barbosa (1994), Tundisi \& Barbosa (1995), Navas-Pereira \& Henrique (1995), Brandimarte \& Shimizu (1996) e Kuhlmann et al. (1998). Entretanto, a maioria dos trabalhos se localiza em represas artificiais, limitando o conhecimento a áreas isoladas e em ecossistemas lênticos.

Os trabalhos desenvolvidos em ecossistemas lóticos muitas vezes não contam com infra-estrutura adequada para análise ambiental, deixando lacunas na implementação de programas de biomonitoramento. As aplicações mais bem-sucedidas são das agências ambientais em conjunto com universidades ou centros de pesquisa, na maioria das vezes importando ou adaptando índices bióticos dos países europeus (Junqueira \& Campos, 1998; Marques \& Barbosa, 2001).

\section{Perspectivas de aplicação no Brasil}

As agências ambientais têm apresentado algumas sugestões para o uso de biomonitoramento, mas a falta de estudos que estabeleçam padrões de coleta, identificação, avaliação e classificação reduz a aplicabilidade dessas metodologias. As agências ambientais estaduais e federal não dispõem de pessoal ou recursos para o estabelecimento desses padrões, devendo esse papel ficar a cargo das universidades e centros de pesquisa. Apesar de sugestões para a criação de um programa nacional de monitoramento das águas (Barbosa, 1994), acreditamos que, devido às dimensões continentais e à diversidade geográfica do Brasil, inicialmente deveriam ser criados comitês técnicos para discutir a padronização de metodologias regionalmente, fornecendo subsídios técnico-científicos às agências ambientais.

De fato, na melhor das hipóteses, o conhecimento é restrito a algumas áreas, localizadas principalmente nas regiões sudeste e sul (Baptista et al., 2001). É necessária, portanto, uma fase de pesquisas "básicas” em bacias hidrográficas, sobretudo nas outras regiões do País (Maltchik \& Medeiros, 2001), com estudos descritivos que estabeleçam a taxonomia e a ecologia das espécies. Como o principal desafio para os países em desenvolvimento reside na necessidade urgente de instalação de programas de biomonitoramento, tanto a etapa "básica" quanto a "aplicada" devem ser realizadas simultaneamente, o que pode acarretar alguns problemas em sua fase inicial, sem no entanto impedir sua aplicação (Buss, 2001).

Um aspecto fundamental a ser considerado em um programa de monitoramento é a habilidade em traduzir a informação tanto para os gestores ambientais quanto para o público em geral. Muitas vezes, a complexidade dos resultados dos métodos tradicionais de avaliação impede a interpretação pelo público leigo, tornando a informação restrita e, por isso, obscura. As ferramentas originadas por programas de biomonitoramento permitem a formação de grupos de monitores ambientais voluntários nas comunidades, que freqüentemente realizam o levantamento de dados com qualidade, sendo considerados nos programas oficiais de monitoramento (Firehock \&West, 1995; Fore et al., 2001). 
A Lei das Águas (Lei 9.433/97) instituiu a Política e o Sistema Nacional de Recursos Hídricos, e institucionalizou a gestão participativa dos diversos níveis - federal, estadual, municipal e local (Muñoz, 2000). Nela a água é reconhecida como bem econômico, sendo determinada a cobrança por seu uso, com as quantias arrecadadas revertidas para a bacia hidrográfica em que foram geradas por determina-

\section{Agradecimentos}

Os autores agradecem aos colegas do Laboratório de Avaliação e Promoção da Saúde Ambiental (Instituto Oswaldo Cruz, Fundação Oswaldo Cruz), do Laboratório de Entomologia do Museu Nacional (Instituto de Biologia, Universidade Federal do Rio de Janeiro) e do Departamento de Entomologia (Universidade do Rio de Janeiro). Este estudo é baseado em parte na Dissertação de Mestrado de Daniel F. Buss (Programa de Pós-Graduação em Ecologia, Universidade Federal do Rio de Janeiro). Esta pesquisa foi apoiada por: Conselho Nacional de Desenvolvimento Científico e Tecnológico, Fundação de Amparo à Pesquisa do Estado do Rio de Janeiro e Fundação Oswaldo Cruz. ção dos Comitês de Bacias. Nesse contexto, os comitês e as Agências de Águas são parceiros poderosos, e recomenda-se que invistam parte desses rendimentos em programas de biomonitoramento. Após uma fase inicial de pesquisas, esses programas reduzem consideravelmente os custos de análise e fornecem informações relevantes para o manejo de bacias hidrográficas.

\section{Referências}

ARMITAGE, P. D., 1995. Behaviour and ecology of adults. In: The Chironomidae: Biology and Ecology of Non-Biting Midges (P. D. Armitage, P. S. Cranston \& L. C. V. Pinder, ed.), pp. 194-224, London: Chapman \& Hall.

ARMITAGE, P. D.; MOSS, D.; WRIGHT, J. F. \& FURSE, M. T., 1983. The performance of a new biological water quality score based on macroinvertebrates over a wide range of unpolluted running-water sites. Water Research, 17:333-347.

BALLOCH, D.; DAVIS, C. E. \& JONES, F. H., 1976. Biological assessment of water quality in three British rivers, the North Esk (Scotland), the Ivel (England) and the Tass (Wales). Water Pollution Control, 75:92-100.

BAPTISTA, D. F.; DORVILLÉ, L. F. M.; BUSS, D. F. \& NESSIMIAN, J. L., 2001. Spatial and temporal organization of aquatic insects assemblages in the longitudinal gradient of a tropical river. Brazilian Journal of Biology, 61:295-304.

BARBOSA, F. A. R. (org.), 1994. Workshop: Brazilian Programme on Conservation and Management of Inland Waters. Acta Limnologica Brasiliensia v. 5. Belo Horizonte: Fundação Biodiversitas/Sociedade Brasileira de Limnologia.

BARBOUR, M. T.; GERRITSEN, J.; GRIFFITH, G. E.; FRYDENBORG, R.; McCARRON, E.; WHITE, J. S. \& BASTIAN, M. L., 1996. A framework for biological criteria for Florida streams using benthic macroinvertebrates. Journal of the North American Benthological Society, 15:185-211. 
BARBOUR, M. T.; GERRITSEN, J.; SNYDER, B. D. \& STRIBLING, J. B., 1999. Rapid Bioassessment Protocols for Use in Streams and Wadeable Rivers: Periphyton, Benthic Macroinvertebrates and Fish. $2^{\text {nd }}$ Ed. Washington, DC: Environmental Protection Agency.

BEST, E. P. H., 1990. Models on metabolism of aquatic weeds and their application potential. In: Aquatic Weeds. The Ecology and Management of Nuisance Aquatic Vegetation (A. H. Pieterse \& K. J. Murphy, ed.), pp. 254-273, Oxford: Oxford University Press.

BOUDOU, A. \& RIBEYRE, F, 1997. Aquatic ecotoxicology: From the ecosystem to the cellular and molecular level. Environmental Health Perspectives, 105:21-35.

BRANDIMARTE, A. L. \& SHIMIZU, G. Y., 1996. Temporal and spatial variations in littoral benthic communities of Paraibuna Reservoir (São Paulo, Brazil). Tropical Ecology, 37:215-222.

BRASIL, 1934. Decreto no 24.643, de 10 de julho de 1934. Decreta o Código de Águas. Coleção de Leis do Brasil, 4:679.

BRASIL, 1986. Resolução CONAMA no 20, de 18 de junho de 1986. Diário Oficial da União, 30 jul.

BRASIL, 1997. Lei no 9.433, de 8 de janeiro de 1997. Institui a Política Nacional de Recursos Hídricos, Cria o Sistema Nacional de Gerenciamento de Recursos Hídricos, e Dá Outras Providêndicas. Diário Oficial da União, 9 jan.

BUIKEMA, A. L. \& VOSHELL, J. R., 1993. Toxicity studies using freshwater benthic macroinvertebrates. In: Freshwater Biomonitoring and Benthic Macroinvertebrates (D. M. Rosenberg \& V. H. Resh, ed.), pp. 344-398, New York: Chappman \& Hall.

BUSS, D. F., 2001. Utilizando Macroinvertebrados no Desenvolvimento de um Procedimento Integrado de Avaliação da Qualidade da Água de Rios. Dissertação de Mestrado, Rio de Janeiro: Programa de Pós-Graduação em Ecologia, Universidade Federal do Rio de Janeiro.

CAIRNS Jr., J.; McCORMICK, P. V. \& NIEDERLEHNER, B. R., 1993. A proposal framework for developing indicators of ecosystem health. Hydrobiologia, 263:1-44.

CAIRNS Jr., J. \& PRATT, J. R., 1993. A history of biological monitoring using benthic macroinvertebrates. In: Freshwater Biomonitoring and Benthic Macroinvertebrates (D. M. Rosenberg \& V. H. Resh, ed.), pp. 10-27, New York: Chapman \& Hall.

CAIRNS Jr., J. \& van der SCHALIE, W. H., 1980. Biological monitoring. Part I - Early warning systems. Water Research, 14:1179-1196.

CALOW, P., 1993. General principles and overview. In: Handbook of Ecotoxicology (P. Calow, ed.), pp. 15, London: Blackwell Scientific Publications.

CNUMAD (Conferência das Nações Unidas sobre Meio Ambiente e Desenvolvimento), 1992. Conferência das Nações Unidas sobre o Meio Ambiente e Desenvolvimento: Agenda 21. Brasília: Senado Federal.

DE PAUW, N. \& VANHOOREN, G., 1983. Method for biological quality assessment of watercourses in Belgium. Hydrobiologia, 100:153-168.

ESTEVES, F. A., 1988. Fundamentos de Limnologia. Rio de Janeiro: Editora Interciência/Financiadora de Estudos e Projetos.
EWELL, W. S.; GORSUCH, J. W.; KRINGLE, R. O.; ROBILLARD, K. A. \& SPIEGEL, R. C., 1986. Simultaneous evaluation of the acute effects of chemicals on seven aquatic species. Environmental Toxicology and Chemistry, 5:831-840.

FAUSCH, K. D.; KARR, J. R. \& YANT, P. R., 1984. Regional application of an index of biotic integrity based on stream fish communities. Transactions of the North American Fisheries Society, 113:3955.

FAUSCH, K. D.; LYONS, J. R.; KARR, J. R. \& ANGERMEIER, P. L., 1990. Fish communities as indicators of environmental degradation. American Fisheries Society Symposium, 8:123-144.

FIREHOCK, K. \& WEST, J., 1995. A brief history of volunteer biological water monitoring using macroinvertebrate. Journal of the North American Benthological Society, 14:197-202.

FORE, L. S.; PAULSEN, K. \& O'LAUGHLIN, K., 2001 Assessing the performance of volunteers in monitoring streams. Freshwater Biology, 46:109-123.

HASLAM, S. M., 1982. A proposed method for monitoring river pollution using macrophytes. Environmental Technology Letters, 3:19-34.

HAWKES, H. A., 1997. Origin and development of the Biological Monitoring Working Party score system. Technical note. Water Research, 32:964-968.

JOHNSON, R. K.; WIEDERHOLM, T. \& ROSENBERG, D. M., 1993. Freshwater biomonitoring using individual organisms, populations, and species assemblages of benthic macroinvertebrates. In: Freshwater Biomonitoring and Benthic Macroinvertebrates (D. M. Rosenberg \& V. H. Resh, ed.), pp. 40-158, New York: Chapman \& Hall.

JUNQUEIRA, V. M. \& CAMPOS, S. C. M., 1998. Adaptation of the "BMWP" method for water quality evaluation to Rio das Velhas watershed (Minas Gerais, Brazil). Acta Limnologica Brasiliensia, 10: 125-135.

KARR, J. R., 1981. Assessment of biotic integrity using fish communities. Fisheries, 6:21-27.

KARR, J. R.; FAUSCH, K. D.; ANGERMEIER, P. L.; YANT, P. R. \& SCHLOSSER, I. J., 1986. Assessment of Biological Integrity in Running Water: A Method and its Rationale. Special Publication 5. Champaign: Illinois Natural History Survey.

KERANS, B. L. \& KARR, J. R., 1994. A benthic index of biotic integrity (B-IBI) for rivers in the Tennessee valley. Ecological Applications, 4:768-785.

KOLKWITZ, R. \& MARSSON, M., 1909. Oekologie der tierischen Saprobien. Internationale Revue der Gesamten Hydrobiologie und Hydrographie, 2: 126-152.

KUHLMANN, M. L.; TRUZZI, A. C. \& FORNASARO, G. J., 1998. The benthos community of the Billings reservoir (São Paulo, Brazil) and its use in environmental quality assessment. Verhandlungen der Internationalen Vereinigung für Theoretische und Angewandte Limnologie, 26:2083-2087.

MALTCHIK, L. \& MEDEIROS, E. S., 2001. Does hydrological stability influence biodiversity and community stability? A theoretical model for lotic ecosystem from Brazilian semiarid region. Ciência e Cultura, 53:44-48.

MARQUES, M. M. \& BARBOSA, F., 2001. Biological quality of waters from an impacted tropical wa- 
tershed (middle Rio Doce basin, southeast Brazil), using benthic macroinvertebrate communities as an indicator. Hydrobiologia, 457:69-76.

MATTHEWS, R. A.; BUIKEMA, A. L. \& CAIRNS Jr., J., 1982. Biological monitoring part IIA: Receiving system functional methods relationships, and indices. Water Research, 16:129-139.

METCALFE, J. L., 1989. Biological water quality assessment of running waters based on macroinvertebrates communities: history and present status in Europe. Environmental Pollution, 60:101139.

MMA (Ministério do Meio Ambiente), 1998. Programa Monitore: Diretório das Instituições que Realizam Monitoramento Ambiental. Brasília: MMA.

MOSS, D.; FURSE, M. T.; WRIGHT, J. F. \& ARMITAGE, P. D., 1987. The prediction of the macroinvertebrate fauna of unpolluted running-water sites in Great Britain using environmental data. Freshwater Biology, 17:41-52.

MOULTON, T. P., 1998. Saúde e integridade do ecossistema e o papel dos insetos aquáticos. In: Ecologia de Insetos Aquáticos (J. L. Nessimian \& A. L. Carvalho, org.), Serie Oecologia Brasiliensis V, pp. 281-298, Rio de Janeiro: Editora UFRJ.

MUÑOZ, H. R. (org.), 2000. Interfaces da Gestão de Recursos Hídricos: Desafios da Lei de Águas de 1997. 2a Ed. Brasília: Secretaria de Recursos Hídricos.

NAVAS-PEREIRA, D. \& HENRIQUES, R. M., 1995. Aplicação de índices biológicos numéricos na avaliação da qualidade ambiental. Revista Brasileira de Biologia, 56:441-450.

OMERNIK, J. M., 1987. Ecoregions of conterminous United States. Annals of the Association of American Geographers, 77:118-125.

PIVETTA, F.; MACHADO, J. M. H.; ARAÚJO, U. C.; MOREIRA, M. F. R. \& APOSTOLI, P., 2001. Monitoramento biológico: Conceitos e aplicações em saúde pública. Cadernos de Saúde Pública, 17:545554.

PLAFKIN, J. L.; BARBOUR, M. T.; PORTER, K. D.; GROSS, S. K. \& HUGHES, R. M., 1989. Rapid Bioassessment Protocols for use in Streams and Rivers: Benthic Macroinvertebrates and Fish. Washington, DC: Environmental Protection Agency.

PRATT, J. M. \& COLER, R. A., 1976. A procedure for the routine biological evaluation of urban runoff in small rivers. Water Research, 10:1019-1025.

RESH, V. H. \& JACKSON, J. K., 1993. Rapid assessment approaches to biomonitoring using benthic macroinvertebrates. In: Freshwater Biomonitoring and Benthic Macroinvertebrates (D. M. Rosenberg \& V. H. Resh, ed.), pp. 195-233, New York: Chapman \& Hall.

REYNOLDSON, T. B. \& METCALFE-SMITH, J. L., 1992. An overview of the assessment of aquatic ecosystem health using benthic invertebrates. Journal of Aquatic Ecosystem Health, 1:295-308.

ROSENBERG, D. M. \& RESH, V. H. (ed.), 1993. Freshwater Biomonitoring and Benthic Macroinvertebrates. New York: Chapman \& Hall.

TUNDISI, J. G. \& BARBOSA, F. A. R., 1995. Conservation of aquatic ecosystems: present status and perspectives. In: Limnology in Brazil (J. G. Tundisi, C. E. M. Bicudo \& T. Matsumura-Tundisi, ed.), pp. 365-376, Rio de Janeiro: Associação Bra- sileira de Ciências/Sociedade Brasileira de Limnologia.

WALLEY, W. J. \& FONTAMA, V. N., 1998. Neural network predictors of Average Score Per Taxon and number of families at unpolluted river sites in Great Britain. Water Research, 32:613-622.

WALLEY, W. J. \& HAWKES, H. A., 1996. A computerbased reappraisal of the Biological Monitoring Working Party scores using data from the 1990 River Quality Survey of England and Wales. Water Research, 30:2086-2094.

WALLEY, W. J. \& HAWKES, H. A., 1997. A computerbased development of the Biological Monitoring Working Party score system incorporating abundance rating, site type and indicator value. Water Research, 31: 201-210.

WASHINGTON, H. G., 1984. Diversity, biotic and similarity indices. A review with special relevance to aquatic ecosystems. Water Research, 18:653-694.

WHITTIER, T. R.; HUGUES, R. M. \& LARSEN, D. P., 1988. Correspondence between ecoregions and spatial patterns in stream ecosystem in Oregon. Canadian Journal of Fisheries and Aquatic Sciences, 45:1264-1278.

WRI (World Resources Institute), 1998. World Resources - 1998-99 - Environmental Change and Human Health. Oxford: Oxford University Press.

WRIGHT, J. F., 1995. Development and use of a system for predicting the macroinvertebrate fauna in flowing waters. Australian Journal of Ecology, 20:181-197.

WRIGHT, J. F.; FURSE, M. T.; ARMITAGE, P. D. \& MOSS, D., 1993. New procedures for identifying running-water sites subject to environmental stress and for evaluating sites for conservation, based on the macroinvertebrate fauna. Archiv für Hydrobiologie, 127:319-326.

WRIGHT, J. F.; MOSS, D.; ARMITAGE, P. D. \& FURSE, M. T., 1984. A preliminary classification of running-water sites in Great Britain based on macroinvertebrate species and the prediction of community type using environmental data. Freshwater Biology, 14:221-256.

Recebido em 17 de junho de 2002

Versão final reapresentada em 16 de setembro de 2002 Aprovado em 19 de novembro de 2002 